PROCEEDINGS OF THE

AMERICAN MATHEMATICAL SOCIETY

Volume 132, Number 11, Pages 3251-3258

S 0002-9939(04)07459-3

Article electronically published on May 12, 2004

\title{
MAPPINGS OF FINITE DISTORTION: REMOVABLE SINGULARITIES FOR LOCALLY HOMEOMORPHIC MAPPINGS
}

\author{
KAI RAJALA
}

(Communicated by Juha M. Heinonen)

\begin{abstract}
Let $f$ be a locally homeomorphic mapping of finite distortion in dimension larger than two. We show that when the distortion of $f$ satisfies a certain subexponential integrability condition, small sets are removable. The smallness is measured by a weighted modulus.
\end{abstract}

\section{Introduction}

We call a mapping $f \in W_{\mathrm{loc}}^{1,1}\left(\Omega, \mathbb{R}^{n}\right)$ a mapping of finite distortion if it satisfies

$$
|D f(x)|^{n} \leq K(x, f) J(x, f) \quad \text { a.e., }
$$

where $K(x, f)<\infty$ and if also $J(\cdot, f) \in L_{\text {loc }}^{1}(\Omega)$. In this paper $\Omega \subseteq \mathbb{R}^{n}$ is an open and connected set. When $K(\cdot, f) \in L^{\infty}(\Omega), f$ is called a mapping of bounded distortion, or a quasiregular mapping. Quasiregular mappings have been widely studied; see the monographs [15] and [16]. Recently there has been an extensive study of mappings of finite distortion. It has especially been shown that, under suitable subexponential integrability conditions on $K(\cdot, f)$, many properties of quasiregular mappings still hold true. See [4, [5], 6], [7] and [8] for some basic properties, such as continuity, discreteness, openness and the Lusin condition. Very recently the important modulus inequalities of quasiregular mappings were generalized to the class of mappings of finite distortion by Koskela and Onninen [10]. These inequalities will be used below in order to prove a removability theorem for locally homeomorphic mappings of finite distortion with sufficiently regular distortion function.

Let us next describe our assumptions on $K(\cdot, f)$. Let $\Phi:[0, \infty) \rightarrow[0, \infty)$ be a strictly increasing, differentiable function. We call such functions Orlicz functions, and we make the following two assumptions:

$(\Phi-1) \int_{1}^{\infty} \frac{\Phi^{\prime}(t)}{t} d t=\infty$

$(\Phi-2) t \Phi^{\prime}(t)$ increases to infinity when $t \rightarrow \infty$.

Received by the editors February 12, 2003 and, in revised form, July 2, 2003.

2000 Mathematics Subject Classification. Primary 30C65.

The author was supported by the foundations Magnus Ehrnroothin Säätiö and Vilho, Yrjö ja Kalle Väisälän Rahasto. A part of this research was done when the author was visiting at the University of Michigan. He wishes to thank the department for its hospitality.

(C)2004 American Mathematical Society 
We shall consider mappings $f$ of finite distortion for which there exists a $\Phi$, satisfying conditions $(\Phi-1)$ and $(\Phi-2)$, such that

$$
\exp (\Phi(K(\cdot, f))) \in L_{l o c}^{1}(\Omega) .
$$

This assumption has turned out to be very sharp; for any Orlicz function that does not satisfy $(\Phi-1)$, one can construct mappings of finite distortion satisfying (1.2) and violating all of the above-mentioned basic properties; see 9]. One of the constructions in [9] maps $B(0,1) \backslash\{0\}$ homeomorphically onto an annulus, showing that this assumption is sharp also for our Theorem 1.1 below.

The size of the removable set in our theorem will be given in terms of a weighted modulus. Recall that the (conformal) $n$-modulus of a path family $\Gamma$ is defined by

$$
\begin{aligned}
M(\Gamma)= & \inf \left\{\int_{\mathbb{R}^{n}} \rho^{n}(x) d x: \rho: \mathbb{R}^{n} \rightarrow[0, \infty)\right. \text { is a Borel function such that } \\
& \left.\int_{\gamma} \rho \geq 1 \text { for each locally rectifiable } \gamma \in \Gamma\right\} .
\end{aligned}
$$

We shall also use the $n$-modulus on spheres. If $S^{n-1}(a, r)$ is a sphere, define the $n$-modulus of a path family $\Gamma$ on $S^{n-1}(a, r)$ by

$$
\begin{aligned}
M^{S}(\Gamma)= & \inf \left\{\int_{S} \rho^{n}(x) d \mathcal{H}^{n-1}(x): \rho: S^{n-1}(a, r) \rightarrow[0, \infty)\right. \text { is a Borel } \\
& \text { function such that } \left.\int_{\gamma} \rho \geq 1 \text { for each locally rectifiable } \gamma \in \Gamma\right\} .
\end{aligned}
$$

For a mapping $f$ of finite distortion, define the $K^{n-1}$-modulus $M_{K^{n-1}(\cdot, f)}(\Gamma)$ by

$$
\begin{array}{r}
M_{K^{n-1}(\cdot, f)}(\Gamma)=\inf \left\{\int_{\mathbb{R}^{n}} \rho^{n}(x) K^{n-1}(x, f) d x: \rho: \mathbb{R}^{n} \rightarrow[0, \infty)\right. \text { is a Borel } \\
\text { function such that } \left.\int_{\gamma} \rho \geq 1 \text { for each locally rectifiable } \gamma \in \Gamma\right\} .
\end{array}
$$

For a set $A \subseteq \mathbb{R}^{n}$, we denote $M_{K^{n-1}(\cdot, f)}(A)=M_{K^{n-1}(\cdot, f)}(\Gamma)$, where $\Gamma$ is the family of all non-constant paths starting at $A$. Note that since $K(x, f) \geq 1$ for all $x \in \Omega$, the inequality $M(\Gamma) \leq M_{K^{n-1}(\cdot, f)}(\Gamma)$ always holds. In particular, sets of zero $K^{n-1}$ modulus have zero Hausdorff dimension. On the other hand, Theorem 5.3 in [10] shows that, under assumption (1.2), singletons have zero $K^{n-1}$-modulus. Hence the removable set in Theorem 1.1 below may always be chosen to be nonempty.

We denote by $\overline{\mathbb{R}}^{n}$ the one-point compactification of $\mathbb{R}^{n}$, and give $\overline{\mathbb{R}}^{n}$ the spherical metric, which is the metric that makes the stereographic projection $\pi: \overline{\mathbb{R}}^{n} \rightarrow$ $S^{n}\left(e_{n+1} / 2,1 / 2\right)$ an isometry. We extend the definition of a mapping of finite distortion to $\overline{\mathbb{R}}^{n}$-valued mappings as follows: Let $f: \Omega \rightarrow \overline{\mathbb{R}}^{n}$ be a mapping, $\Omega \subseteq \mathbb{R}^{n}$. Then $f$ is a mapping of finite distortion if each $x \in \Omega$ has a neighborhood $U \subseteq \Omega$ so that $g \circ f_{U}: U \rightarrow \mathbb{R}^{n}$ is a mapping of finite distortion for some Möbius transformation $g: \overline{\mathbb{R}}^{n} \backslash\{p\} \rightarrow \mathbb{R}^{n}$, where $f_{U}$ is the restriction of $f$ to $U$. Note that assumption (1.2) is well-defined for $\overline{\mathbb{R}}^{n}$-valued mappings also, since the distortion does not depend on the Möbius transformation $g$. In the case of quasiregular mappings, the $\overline{\mathbb{R}}^{n}$-valued generalizations are often called quasimeromorphic mappings. Although the target space in Theorem 1.1 is $\overline{\mathbb{R}}^{n}$, we shall only use Euclidean distances in the target side, since our considerations will be local. The compactified space is needed, however, since we will compose our mapping with Möbius transformations, which possibly map infinity to a finite point. 
We are now ready to state our theorem.

Theorem 1.1. Assume $n \geq 3$. Let $f: \Omega \backslash E \rightarrow \overline{\mathbb{R}}^{n}$ be a locally homeomorphic mapping of finite distortion, and assume that $E$ is a compact set for which $M_{K^{n-1}(\cdot, f)}(E)=0$. Moreover, assume that (1.2) holds with $\Phi$ satisfying conditions $(\Phi-1)$ and $(\Phi-2)$. Then $f$ extends to a locally homeomorphic mapping of finite distortion $\tilde{f}: \Omega \rightarrow \overline{\mathbb{R}}^{n}$ satisfying (1.2).

Theorem 1.1 generalizes Dairbekov's result for quasiregular mappings; see [2]. Earlier related papers are [1] by Agard and Marden, and [18] by Zorich. Our proof uses the ideas of these authors and the modulus inequalities of [10].

Earlier removability results for mappings of finite distortion were proved in [3] and 11], using different methods. In particular, it was shown in [11] that if a given $\Phi$ does not satisfy assumption $(\Phi-1)$, then there exists a bounded mapping $f$ of finite distortion, defined in the punctured unit cube $Q_{1} \backslash\{0\}$, so that $f$ satisfies assumption (1.2) but does not extend to a mapping of finite distortion of the unit cube satisfying (1.2).

We also have the following removability theorem: If $f$ is as in Theorem 1.1, but not necessarily locally homeomorphic, and if $f$ omits a set of positive conformal modulus, then sets of zero $K^{n-1}$-modulus are removable. This is a generalization of a theorem by Martio, Rickman and Väisälä ([13], Theorem 4.1), and their proof can essentially be carried out also in our situation, if we replace the modulus inequalities and modulus of continuity estimates of quasiregular mappings by the corresponding ones for mappings of finite distortion; see [10, [5].

\section{Proof of Theorem 1.1}

We first observe that the main task in proving Theorem 1.1 is to prove the existence of a locally homeomorphic extension.

Lemma 2.1. Let $f$ and $E$ be as in Theorem 1.1. If $f$ has a locally homeomorphic extension $\tilde{f}$ to $\Omega$, then $\tilde{f}$ is a mapping of finite distortion satisfying (1.2).

Proof. We may assume that $\tilde{f}$ is $\mathbb{R}^{n}$-valued. Since $E$ has Hausdorff dimension zero, the coordinate functions of $\tilde{f}$ are absolutely continuous on almost every line parallel to the coordinate axis (ACL), and thus weak partial derivatives exist. By using inequality (1.1), the subexponential integrability of $K(\cdot, f)$ and Hölder's inequality, one can see that local integrability of the Jacobian of $\tilde{f}$ implies local integrability of the weak partial derivatives. So it suffices to show that the Jacobian of $\tilde{f}$ is locally integrable.

Let $A \subseteq \Omega$ be a compact set. Now for the locally homeomorphic mapping $\tilde{f}$, the multiplicity function $N(\cdot, A)$ is bounded by some $M<\infty$. On the other hand, the ACL property of $\tilde{f}$, together with the Lusin condition, implies that the area formula holds; see [12], Theorem 9.2. Thus

$$
\begin{aligned}
\int_{A} J_{\tilde{f}} & =\int_{A \backslash E} J_{f}=\int_{\mathbb{R}^{n}} N(y, A \backslash E) d y \\
& =\int_{f(A)} N(y, A \backslash E) d y \leq M|f(A)|<\infty .
\end{aligned}
$$


We will need the following properties of local homeomorphisms; see [14 for proofs.

A set $A \subseteq \mathbb{R}^{n}$ is called relatively locally connected, if every point in $\bar{A}$ has arbitrarily small neighborhoods $U$ such that $U \cap A$ is connected. Note that the punctured sphere $S^{n-1} \backslash\{p\}$ is relatively locally connected if and only if $n \geq 3$.

Lemma 2.2. Let $f: G \rightarrow \overline{\mathbb{R}}^{n}$ be a local homeomorphism. Let $A \subseteq \overline{\mathbb{R}}^{n}$ be simply connected and locally pathwise connected, and let $P$ be a component of $f^{-1}(A)$ such that $\bar{P} \subseteq G$. Then $f$ maps $P$ homeomorphically onto $A$. If, in addition, $A$ is relatively locally connected, then $f$ maps $\bar{P}$ homeomorphically onto $\bar{A}$.

Lemma 2.3. Let $f: G \rightarrow \overline{\mathbb{R}}^{n}$ be a local homeomorphism, and let $F$ be a compact set in $G$ such that $f$ is injective in $F$. Then $f$ is injective in a neighborhood of $F$.

Lemma 2.4. Let $f: G \rightarrow \overline{\mathbb{R}}^{n}$ be a local homeomorphism, let $A, B \subseteq G$, and let $f$ be homeomorphic in both $A$ and $B$. If $A \cap B \neq \emptyset$ and if $f(A) \cap f(B)$ is connected, then $f$ is homeomorphic in $A \cup B$.

Proof of Theorem 1.1. By Lemma 2.1 it suffices to show that $f$ has a locally homeo-

morphic extension to $\Omega$. Consider the minimal subset of $E$ with the property that $f$ does not have a locally homeomorphic extension to any point in that set; it is clear that the minimal set exists. Without loss of generality, we may assume that $E$ is this set. We are going to show that, for some point $z \in E, f$ extends to a neighborhood of $z$ as a local homeomorphism, which forces $E$ to be the empty set. We may assume that $0 \in E, B(0,1) \subseteq \Omega$ and $S(0,1) \cap E=\emptyset$.

The following modulus inequality, which is a counterpart of the Poletsky inequality of quasiregular mappings, is a special case of Theorem 4.1 in [10].

Lemma 2.5. Let $f: \Omega \rightarrow \mathbb{R}^{n}$ be a mapping of finite distortion satisfying (1.2), with an Orlicz function $\Phi$ for which $(\Phi-1)$ and $(\Phi-2)$ hold. Let $\Gamma$ be a path family in $\Omega$. Then

$$
M(f \Gamma) \leq M_{K^{n-1}(\cdot, f)}(\Gamma) .
$$

Using Lemma 2.5 we have the following modification of [1], Step 1.

Lemma 2.6. Let $f$ and $E$ be as above. Let $a \in \mathbb{R}^{n}, 0 \leq r_{1}<r_{2}$, and let $L$ be a ray emanating from a. Assume that $S(a, r) \cap f(S(0,1))=\emptyset$ for all $r \in\left(r_{1}, r_{2}\right)$. Moreover, assume that a local inverse $f_{0}^{-1}: U \rightarrow B(0,1) \backslash E$ of $f$ is specified in a neighborhood $U$ of $I:=\left\{x \in L:|x-a| \in\left(r_{1}, r_{2}\right)\right\}$. Then, for almost every $r \in\left(r_{1}, r_{2}\right), f_{0}^{-1}$ can be extended from $S(a, r) \cap U$ to a homeomorphism (we denote the extension also by $\left.f_{0}^{-1}\right)$ of $S(a, r)$ onto $f_{0}^{-1}(S(a, r))=: S_{r}^{*} \subseteq B(0,1) \backslash E$. Furthermore, for each such $r$ the following holds: if $\operatorname{int}\left(S_{r}^{*}\right)=: B_{r}^{*} \subseteq B(0,1) \backslash E$, then $f_{0}^{-1}$ can be extended from $S(a, r)$ to a homeomorphism of either $\operatorname{int}(S(a, r))$ or $\operatorname{ext}(S(a, r))$ onto $B_{r}^{*}$. Here and in what follows the notation ext is used to denote the component of the complement of the topological sphere that contains infinity, while the notation int is used for the other component.

Proof. For each $r \in\left(r_{1}, r_{2}\right)$, consider the spherical caps $C_{s, r}$ with center $a$ and radius $r$, opening from the unique point $k_{r}$ in $S(a, r) \cap I$, with the opening angle $s$. Let $t(r)$ be the supremum of those opening angles $s$ for which $f_{0}^{-1}$ extends from 
$U \cap C_{s, r}$ to $C_{s, r}$ as a homeomorphism. Now, by Lemma 2.2, either $f_{0}^{-1}$ extends to $\bar{C}_{t(r), r}$ as a homeomorphism, or

$$
\partial f_{0}^{-1}\left(C_{t(r), r}\right) \cap E \neq \emptyset .
$$

Note that we assumed $S(a, r) \cap f(S(0,1))=\emptyset$. Lemma 2.3 now implies that in the former case $t(r)=\pi$, and hence by Lemma 2.2 $f_{0}^{-1}$ extends to a homeomorphism of $S(a, r)$ in the former case. In the latter case there exists $y(r) \in \partial C_{t(r), r}$ such that every path $\gamma$ in $\bar{C}_{t(r), r}$ connecting $k_{r}$ to $y(r)$ has a lift $\gamma^{\prime}$ so that $\overline{\left|\gamma^{\prime}\right|} \cap E \neq \emptyset$. To show this, consider for a $y \in \partial C_{t(r), r}$ a path $\gamma$ connecting $k_{r}$ to $y$ on the cap. Then for the unique lift $\gamma^{\prime}$ of $\gamma, y^{\prime} \in \overline{\left|\gamma^{\prime}\right|}$ for a point $y^{\prime}$ in $\partial f_{0}^{-1}\left(C_{t(r), r}\right)$. Now, if $y^{\prime} \in \Omega \backslash E$, then $f$ is a local homeomorphism mapping a neighborhood of $y^{\prime}$ onto a neighborhood of $y$. Moreover, by Lemma 2.4, the inverse around $y$ is an extension of $f_{0}^{-1}$. If this happens for all points on the boundary of the cap, then $f_{0}^{-1}$ extends to the boundary as a homeomorphism, which is a contradiction by Lemma 2.3 and the maximality of the homeomorphic extension.

We want to show that the set of the radii for which the latter case applies has linear measure zero. Denote this set by $A$. For $r \in A$, let $\Gamma_{r}$ be the set of all paths joining $k_{r}$ to $y(r)$ in $C_{t(r), r}$. Then by [17], Theorem 10.2,

$$
M^{S}\left(\Gamma_{r}\right) \geq \frac{C_{n}}{r} .
$$

If we denote $\Gamma=\bigcup_{r \in A} \Gamma_{r}$, then (2.2) gives

$$
M(\Gamma) \geq C_{n} \int_{A} \frac{1}{r} d r .
$$

We assumed that each path in $\Gamma$ has a lift ending at some point of $E$. Thus there exists a path family $\Gamma^{\prime}$ such that $f\left(\Gamma^{\prime}\right)=\Gamma$ and $\Gamma^{\prime} \subseteq \Gamma^{*}=\{\gamma: \gamma$ is a path ending at $E\}$. Since $M_{K^{n-1}(\cdot, f)}\left(\Gamma^{*}\right)=0$, we have, by Lemma 2.5 and inequality (2.3),

$$
C_{n} \int_{A} \frac{1}{r} \leq M(\Gamma) \leq M_{K^{n-1}(\cdot, f)}\left(\Gamma^{\prime}\right) \leq M_{K^{n-1}(\cdot, f)}\left(\Gamma^{*}\right)=0,
$$

and thus $|A|=0$, as desired.

The latter claim of the lemma is proved by the same argument as in [1, page 458.

By composing $f$ with a Möbius transformation if necessary, we may assume that there exists a sequence $\left(x_{n}\right)$ in $B(0,1) \backslash E$ such that $x_{n} \rightarrow 0$ and $y_{n}=f\left(x_{n}\right) \rightarrow$ 0 . We may also assume that $0 \notin f(S(0,1))$ and that $\left|y_{n}\right|<d(0, f(S(0,1)))$ for all $n$. Now, for each $n$, there exists a neighborhood $U_{n}$ of $y_{n}$ such that $U_{n} \subseteq$ $B(0, d(0, f(S(0,1))))$ and that the local inverse $f_{n}^{-1}$ of $f$, determined by $f_{n}^{-1}\left(y_{n}\right)=$ $x_{n}$, is a homeomorphism of $U_{n}$ onto some $U_{n}^{\prime}$ containing $x_{n}$. We may also assume that $\sup _{x \in U_{n}^{\prime}}|x| \rightarrow 0$.

Let $L_{n}$ be a ray starting at 0 and passing through $y_{n}$. Lemma 2.6 then implies that there exists a sphere $S_{n}=S\left(0, r_{n}\right)$ intersecting $U_{n}$ so that $S_{n}^{\prime}=f_{n}^{-1}\left(S_{n}\right)$ is a topological sphere in $B(0,1) \backslash E$, intersecting $U_{n}^{\prime}$. As previously, we denote the extension of the local inverse also by $f_{n}^{-1}$. Note that $r_{n} \rightarrow 0$.

Denote $B_{n}^{*}=\operatorname{int}\left(S_{n}^{\prime}\right)$. Let us first assume that for some index $n$,

$$
B_{n}^{*} \cap E \neq \emptyset .
$$


For each $q \in S_{n}$, consider the line segment $L_{q}^{s}=\{t q: t \in(s, 1]\}$. Define $L_{q}$ to be the maximal $L_{q}^{s}$ for which $f_{n}^{-1}$ is a homeomorphism from $L_{q}$ onto the lift $L_{q}^{\prime}$ that starts at $f_{n}^{-1}(q)$. Since $S_{n}^{\prime} \subseteq B(0,1)$ and $B_{n} \cap f(S(0,1))=\emptyset\left(B_{n}=B\left(0, r_{n}\right)\right)$, it follows from Lemma 2.2 that for all the $L_{q}$ 's that do not reach the origin, the lift $L_{q}^{\prime}$ must end at some point in $E$. Since $M_{K^{n-1}(\cdot, f)}(E)=0$, inequality (2.1) implies that, for almost all $q \in S_{n}, L_{q}$ reaches the origin. Denote the set of these $q$ 's by $Z$ and fix one $q \in Z$.

Now we apply Lemma 2.6 again in order to show that, for almost all points $p$ in $L_{q}$ with respect to the Hausdorff 1-measure, the mapping $f$ has an inverse from $S_{p}=S(0,|p|)$ onto some $S_{p}^{\prime} \subseteq B(0,1)$. Denote the set of those $p$ by $T$. Lemma 2.4 now implies that if we denote

$$
W=\left(\bigcup_{q \in Z} L_{q}\right) \cup\left(\bigcup_{p \in T} S_{p}\right)
$$

and

$$
W^{\prime}=\left(\bigcup_{q \in Z} L_{q}^{\prime}\right) \cup\left(\bigcup_{p \in T} S_{p}^{\prime}\right)
$$

then in fact $f_{n}^{-1}: W \rightarrow W^{\prime}$ is a homeomorphism. We claim that $f_{n}^{-1}$ actually extends to a homeomorphism of $B_{n} \backslash\{0\}$. To prove the claim, we modify part of the proof of Lemma 2.2 given in [14].

Since $Z \subseteq S_{n}$ and $T \subseteq L_{q}$ are dense, $W$ is relatively locally connected. Let $y \in \bar{W}$. We want to show that there exists a limit $f_{n}^{-1}(y)$. Choose neighborhoods $V_{1} \supseteq V_{2} \supseteq \ldots$ so that $\bigcap_{1}^{\infty} V_{i}=\{y\}$ and $V_{i} \cap W$ is connected for all $i$. Then also $f_{n}^{-1}\left(V_{i} \cap W\right)$ is connected for all $i$, and

$$
\left.\overline{\left(f_{n}^{-1}\left(V_{i} \cap W\right)\right.}\right)_{i}
$$

is a nested sequence of connected compact sets. Thus

$$
Y=\bigcap_{i=1}^{\infty} \overline{f_{n}^{-1}\left(V_{i} \cap W\right)}
$$

is connected and nonempty. On the other hand, $Y \subseteq f^{-1}(y) \cup E$. Since $f$ is discrete and $E$ has zero Hausdorff dimension, it follows that $Y$ is a point. This proves the claim.

Now the boundary of $f_{n}^{-1}\left(B_{n} \backslash\{0\}\right)$ has two components. Since $f_{n}^{-1}$ is a homeomorphism in $B_{n} \backslash\{0\}$, one of the components has the property that for every path $\gamma$ in $B_{n} \backslash\{0\}$ ending at the origin, the lift $\gamma^{\prime}$ ends at this component. Since $E$ has Hausdorff dimension zero, the local homeomorphism property of $f$ is then violated on points of this component, unless the component is a point. Recall that $f_{n}^{-1}\left(B_{n} \backslash\{0\}\right) \subset B(0,1)$. Thus $f_{n}^{-1}$ extends to a homeomorphism of $B_{n}$, and $f_{n}^{-1}\left(B_{n}\right)=B_{n}^{*}$. This contradicts (2.4), unless $E$ is empty.

Let us then assume that for all spheres $S_{n}$,

$$
B_{n}^{*} \cap E=\emptyset .
$$

Then by Lemma 2.6, $f_{n}^{-1}$ extends, for all $n$, to a homeomorphism of either $B\left(0, r_{n}\right)$ $=B_{n}$ onto $B_{n}^{*}$, or $\overline{\mathbb{R}}^{n} \backslash B_{n}$ onto $B_{n}^{*}$. By taking a subsequence, we may assume that the same case occurs for all $n$. The two cases are similar, and thus we will only consider the former.

Let $t_{n}$ be the supremum of numbers $s \geq r_{n}$ with the property that $f_{n}^{-1}$ has a homeomorphic extension to $B_{s}=B(0, s)$, mapping into $B(0,1) \backslash E$. We then claim 
that $t_{n}<\infty$ and that $f_{n}^{-1}$ has an extension to $\bar{B}_{t_{n}}$, mapping into $\bar{B}(0,1) \backslash E$, so that $f_{n}^{-1}\left(S_{t_{n}}\right)$ intersects $S(0,1)$.

To prove the first part of the claim, we assume that $t_{n}=\infty$. We have shown above that a local inverse of $f$, mapping from a punctured ball, extends to the whole ball. The same argument shows that $f_{n}^{-1}$ has a limit at $\infty$, and thus $f_{n}^{-1}$ extends to a homeomorphism mapping $\overline{\mathbb{R}}^{n}$ into $\bar{B}(0,1) \backslash E$, which is topologically impossible. Thus $t_{n}<\infty$.

To prove the second part of the claim, set $S_{t_{n}}^{*}=f_{n}^{-1}\left(S_{t_{n}}\right)$ and assume that $f_{n}^{-1}$ does not extend to $\bar{B}_{t_{n}}$. It follows that there exist points $z_{0} \in E$ and $y_{0} \in S_{t_{n}}$, and a sequence $\left(y_{\eta}\right) \subseteq B\left(0, t_{n}\right)$ such that $y_{\eta} \rightarrow y_{0}$ and $f_{n}^{-1}\left(y_{\eta}\right) \rightarrow z_{0}$. As previously, we find a sequence of spheres $S\left(y_{0}, t_{\eta}\right)=S_{\eta}$ intersecting a small neighborhood of $y_{\eta}$, so that the spheres shrink to $y_{0}$ and $f_{n}^{-1}$ extends to $B_{t_{n}} \cup S_{\eta}$ as a homeomorphism. We may assume that $E \cap \operatorname{int}\left(f_{n}^{-1}\left(S_{\eta}\right)\right)=\emptyset$; otherwise the case (2.4) applies. By Lemma 2.6, $f_{n}^{-1}$ extends as a homeomorphism mapping either $\operatorname{int}\left(S_{\eta}\right)$ or $\operatorname{ext}\left(S_{\eta}\right)$ onto $\operatorname{int}\left(f_{n}^{-1}\left(S_{\eta}\right)\right)$. The former case is not possible, however, since $f_{n}^{-1}\left(y_{\eta}\right) \rightarrow z_{0} \in E$. Thus $f_{n}^{-1}$ maps $\operatorname{ext}\left(S_{\eta}\right) \cup B_{t_{n}}$ into $\bar{B}(0,1) \backslash E$. Letting $\eta \rightarrow \infty$, we see that $f_{n}^{-1}$ extends to a homeomorphism mapping $\overline{\mathbb{R}}^{n} \backslash\left\{y_{0}\right\}$ into $\bar{B}(0,1) \backslash E$, and furthermore, $f_{n}^{-1}$ also has a limit at $y_{0}$. This is topologically impossible. We have shown that $f_{n}^{-1}$ extends to $\bar{B}_{t_{n}}$, mapping into $\bar{B}(0,1) \backslash E$. By the definition and the finiteness of $t_{n}$ and Lemma 2.3 it follows that $f_{n}^{-1}\left(S_{t_{n}}\right)$ intersects $S(0,1)$. Thus the other part of the claim holds true.

We have constructed a sequence of balls $B_{t_{n}}$ so that $f_{n}^{-1}\left(B_{t_{n}}\right)=S_{t_{n}}^{*}$ intersects $S(0,1)$ and $f_{n}^{-1}$ is a homeomorphism mapping $\bar{B}_{t_{n}}$ onto $\bar{B}_{t_{n}}^{*}=\operatorname{int}\left(S_{t_{n}}^{*}\right) \cup S_{t_{n}}^{*}$. Now, if two $\bar{B}_{t_{n}}^{*}$ 's intersect, they have to be identical, which is seen as follows. By construction, the mappings $f_{n}^{-1}$ for $n \in \mathbb{N}$ are different branches of $f^{-1}$, defined at balls centered at the origin. Then, if two $\bar{B}_{t_{n}}^{*}$ 's intersect, Lemma 2.4 implies that there exists an inverse defined in their union, which is a contradiction. Recall that $f_{n}^{-1}$ was determined by the requirement $f_{n}^{-1}\left(y_{n}\right)=x_{n}$ and that each $S_{n}^{*}=f_{n}^{-1}\left(S_{n}\right)$ passes through a neighborhood $U_{n}^{\prime}$ of $x_{n}$ that tends to zero as $n \rightarrow \infty$. Since $S_{n}^{*} \subseteq \bar{B}_{t_{n}}^{*}$, we conclude that $B_{t_{m}}^{*} \cap B_{t_{n}}^{*}=\emptyset$ for infinitely many indices, and we may assume this to be true for all of them.

We may also assume that there exists a sequence $\left(x_{n}^{\prime}\right)$ such that $x_{n}^{\prime} \in S_{t_{n}}^{*} \cap S(0,1)$ and $x_{n}^{\prime} \rightarrow x_{0}^{\prime} \in S(0,1)$. Recall that $S(0,1) \cap E=\emptyset$. Therefore $f\left(x_{n}^{\prime}\right) \rightarrow f\left(x_{0}^{\prime}\right)=: y_{0}^{\prime}$. The requirement $f_{0}^{-1}\left(y_{0}^{\prime}\right)=x_{0}^{\prime}$ uniquely determines a local inverse $f_{0}^{-1}$ of $f$ in a neighborhood $U$ of $y_{0}^{\prime}$. Now $U \cap S_{t_{n}} \neq \emptyset$ for all $n>N$, when $N$ is big enough. Hence for all $n>N, f_{n}^{-1}$ is by Lemma 2.4 an extension of $f_{0}^{-1}$, which is a contradiction. This proves the theorem.

\section{ACKNOWLEDGEMENTS}

The author wishes to express his thanks to Professor Pekka Koskela for several suggestions and for reading the manuscript.

\section{REFERENCES}

[1] S. Agard and A. Marden: A removable singularity theorem for local homeomorphisms, Indiana Univ. Math. J., 20 (1970), 455-461. MR 43:2209

[2] N. S. Dairbekov: Removable singularities of locally quasiconformal mappings, Siberian Math. J., 33 (1992), no. 1, 159-161. MR 93d:30029 
[3] T. Iwaniec, P. Koskela, G. Martin, and C. Sbordone: Mappings of finite distortion: $L^{n} \log ^{\chi} L$ integrability, J. London Math. Soc. (2), 67 (2003), no. 1, 123-136. MR 2003h:30031

[4] T. Iwaniec, P. Koskela, and J. Onninen: Mappings of finite distortion: Monotonicity and continuity, Invent. Math. 44 (2001), no. 3, 507-531. MR 2002c:30029

[5] T. Iwaniec, P. Koskela, and J. Onninen: Mappings of finite distortion: Compactness, Ann. Acad. Sci. Fenn. Math., 27 (2002), no. 2, 391-417. MR 2004b:30045

[6] T. Iwaniec and G. Martin: Geometric Function Theory and Nonlinear Analysis, Oxford Mathematical Monographs, The Clarendon Press, Oxford University Press, New York, 2001. MR 2003c:30001

[7] J. Kauhanen, P. Koskela, and J. Malý: Mappings of finite distortion: Discreteness and openness, Arch. Rational Mech. Anal., 160 (2001), 135-151. MR 2002m:30023

[8] J. Kauhanen, P. Koskela, and J. Malý: Mappings of finite distortion: Condition N, Michigan Math. J., 49 (2001), no. 1, 169-181. MR 2002d:30027

[9] J. Kauhanen, P. Koskela, J. Malý, J. Onninen, and X. Zhong: Mappings of finite distortion: Sharp Orlicz-conditions, Preprint 239, Department of Mathematics and Statistics, University of Jyväskylä, 2001.

[10] P. Koskela and J. Onninen: Mappings of finite distortion: Capacity and modulus inequalities, Preprint 257, Department of Mathematics and Statistics, University of Jyväskylä, 2002.

[11] P. Koskela and K. Rajala: Mappings of finite distortion: Removable singularities, Israel J. Math., 136 (2003), 269-283.

[12] J. Malý: Lectures on change of variables in integral, Graduate School in Helsinki, 2001.

[13] O. Martio, S. Rickman, and J. Väisälä: Distortion and singularities of quasiregular mappings, Ann. Acad. Sci. Fenn. Ser. A I Math., 465 (1970), 1-13. MR 42:1995

[14] O. Martio, S. Rickman, and J. Väisälä: Topological and metric properties of quasiregular mappings, Ann. Acad. Sci. Fenn. Ser. A I Math., 488 (1971), 1-31. MR 45:8830

[15] Yu. G. Reshetnyak: Space Mappings with Bounded Distortion, Transl. of Mathematical Monographs, vol. 73, Amer. Math. Soc., Providence, RI, 1989. MR 90d:30067

[16] S. Rickman: Quasiregular mappings, Ergebnisse der Mathematik und ihrer Grenzgebiete (3) [Results in Mathematics and Related Areas (3)], 26, Springer-Verlag, Berlin, 1993. MR 95g:30026

[17] J. Väisälä: Lectures on n-dimensional quasiconformal mappings, Springer-Verlag, Berlin and New York, 1971. MR 56:12260

[18] V. A. Zorich: Isolated singularity of mappings with bounded distortion, Mat. Sb., 81 (123) (1970), no. 4, 634-636; English transl., Math USSR-Sb., 10 (1970), 581-583. MR 42:497

University of Jyväskylä, Department of Mathematics and Statistics, P.O. Box 35, FIN-40014 University of Jyväskylä, Finland

E-mail address: kirajala@maths.jyu.fi 